\title{
Milled legume grain as urease source for the ammonization of elephant grass hay
}

\author{
Leonardo Fiusa de Morais(1), João Carlos de Carvalho Almeida(1), Delci de Deus Nepomuceno(1), \\ Mirton José Frota Morenz ${ }^{(2)}$, Bárbara Maria Gomes de Melo ${ }^{(1)}$ and Rafaela Scalise Xavier de Freitas ${ }^{(1)}$
}

(1)Universidade Federal Rural do Rio de Janeiro, BR 465, Km 7, CEP 23891-000 Seropédica, RJ, Brazil. E-mail: leonardofiusa@yahoo.com.br, delci_ufrrj@yahoo.com.br, joaocarlosbq@gmail.com, barbara.maria.zootecnia@gmail.com, rafascalise@hotmail.com (2)Embrapa Gado de leite, Rua Eugênio do Nascimento, Dom Bosco, no 610, CEP 36038-330 Juiz de Fora, MG, Brazil. E-mail: mirton.morenz@embrapa.br

\begin{abstract}
The objective of this work was to evaluate the use of soybean (Glycine max), pigeon pea (Cajanus cajan), and jack bean (Canavalia ensiformis) as urease sources for elephant grass (Pennisetum purpureum) hay ammoniated with urea. The experimental design was completely randomized in a double factorial arrangement with one additional treatment: 4 urease source levels x 3 urease sources +1 control. Chemicalbromatological analyses and carbohydrate fractionation were performed in the hay, and cumulative gas production in vitro was determined. There were interactions between urease level and source for neutral detergent fiber and acid detergent fiber, in which 1 and $2 \%$ jack bean lowered acid detergent fiber values, and $2 \%$ jack bean and 3\% soybean reduced lignin content. The addition of milled legume grains reduces fiber components and increases non protein nitrogen content in elephant grass hay ammoniated with urea. Adding $4 \%$ milled soybean increases gas production in the soluble fraction.

Index terms: Cajanus cajan, Canavalia ensiformis, Pennisetum purpureum, non-protein nitrogen, roughage.

\section{Grãos de leguminosas moídas como fonte de urease para amonização do feno de capim-elefante}

\begin{abstract}
Resumo - O objetivo deste trabalho foi avaliar o efeito da inclusão de soja (Glycine max), feijão-guandu (Cajanus cajan) e feijão-de-porco (Canavalia ensiformis) como fonte de urease para feno de capim-elefante (Pennisetum purpureum) amonizado com ureia. O delineamento experimental foi inteiramente casualizado, em arranjo fatorial duplo, com um tratamento adicional: 4 níveis de fonte de urease $\mathrm{x} 3$ fontes de urease +1 controle. Realizou-se a análise química-bromatológica e o fracionamento de carboidratos no feno, e a produção cumulativa de gás in vitro foi determinada. Houve efeito da interação entre o nível e a fonte de urease para fibra em detergente neutro e fibra em detergente ácido, em que a adição de 1 e $2 \%$ de feijão-deporco resultou nos menores valores de fibra em detergente ácido, e a adição de $2 \%$ de feijão-de-porco e $3 \%$ de soja, nos menores valores de lignina. A adição de grãos moídos das leguminosas reduz os componentes da fibra e aumenta o teor de nitrogênio não proteico do feno de capim-elefante amonizado com ureia. A adição de 4\% de grão de soja moído aumenta a produção de gás proveniente da fração solúvel.
\end{abstract}

Termos para indexação: Cajanus cajan, Canavalia ensiformis, Pennisetum purpureum, nitrogênio não proteico, volumosos.

\section{Introduction}

Ammonization of low-quality forage with urea is cheap and accessible for farmers (Vadiveloo \& Fadel, 2009). Urea has advantages over anhydrous ammonia, such as greater commercial availability and transportability.
Moisture and urea activity in forage interfere with ammonization efficiency and, consequently, with ammonia production (Ramírez et al., 2007). Ammonia reacts with water to form ammonium hydroxide, which degrades ester links between lignin, cellulose, and hemicellulose; increases the access of ruminal 
microorganisms to carbohydrates; and improves digestibility by changing fiber structure (Wanapat et al., 2009; Polyorach \& Wanapat, 2015).

Soybean (Medeiros-Silva et al., 2014) and jack bean (Piovesan et al., 2014) are common urease sources. Other legumes like pigeon pea, however, also contain this enzyme (Balasubramanian et al., 2013).

In ruminant production, urease sources are added to roughage subjected to urea ammonization for the purpose of increasing ammonia release. As urease sources for forage treatments, Sarmento et al. (2001) and Carvalho et al. (2007) cited soybean, and Bertipaglia et al. (2005), Brachiaria decumbens hay, Pennisetum purpureum, and Leucaena leucocephala. However, these authors did not refer to either jack bean or pigeon pea for this use. In addition, little information is available on the costs of adding urease sources in the treatment of forage ammoniated with urea, on the length of the treatment period, or on its effects on forage dry matter content.

The objective of this work was to evaluate the use of soybean, pigeon pea, and jack bean as urease sources for elephant grass (Pennisetum purpureum) hay ammoniated with urea.

\section{Materials and Methods}

The experiment was conducted at the Instituto de Zootecnia of Universidade Federal Rural do Rio de Janeiro, located in the municipality of Seropédica, in the state of Rio de Janeiro $\left(22^{\circ} 46^{\prime} 59^{\prime \prime} \mathrm{S}, 43^{\circ} 40^{\prime} 45^{\prime \prime} \mathrm{W}\right.$, at $33 \mathrm{~m}$ of altitude). The experimental procedures were approved by the research ethics committee, process No. 23083.010666/2014-11.

Two factors were tested: urease sources (soybean, jack bean, and pigeon pea) and their concentrations (equivalent to $1,2,3$, and $4 \%$ dry matter), including a control (no urease source). The experimental design was completely randomized in a factorial arrangement with four replicates.

Elephant grass (Pennisetum purpureum Schumach. 'Cameron') was grown as a hay source. It was harvested, chopped, and converted to hay at flowering after five months of regrowth on a $400-\mathrm{m}^{2}$ area. Hay samples were set aside and analyzed for chemical composition (Table 1), and then the material was sent to a laboratory for ammonization.
The ammonization process was done by placing $500 \mathrm{~g}$ hay in $15-\mathrm{kg}$ pails, adding $4 \% \mathrm{w} / \mathrm{v}$ aqueous urea, which is enough to reduce the dry matter to $70 \%$. Simultaneously, one of the following urease sources was added to the hay-urea mixture: soybean (Glycine max), pigeon pea (Cajanus cajan), or jack bean (Canavalia ensiformis). These were milled and added to the hay at the rates of $1,2,3$, and $4 \%$ dry matter.

The chemical compositions and the urease activity indices of the milled legume seeds were determined according to the methodology described by Gomes \& Oliveira (2011). The obtained results are shown in Table 2.

After the additions of the urea suspension with milled grains, the pails were hermetically sealed for 30 days. The lids were then removed, and the pails were left open for 48 hours to eliminate excess ammonia. The ammoniated material free of milled grains was then sampled for chemical analysis. The samples were dried in a forced-air circulation oven at $55^{\circ} \mathrm{C}$ for 72 hours and ground in Wiley mills to 1-mm particle size (Silva \& Queiroz, 2002).

Dry matter, ash, ether extract, and total nitrogen were determined according to the 934.01, 924.05, 960.39, and 984.13 methods of the Association of Official Analytical Chemists, respectively (Helrich, 1990). Neutral detergent fiber (NDF), acid detergent fiber (ADF), lignin, and cellulose were evaluated according to the methodology proposed by Van Soest et al. (1991). Hemicellulose was derived from the difference between the NDF and ADF contents. Soluble non protein nitrogen (NPN) was obtained using the difference between the amount of precipitated protein in trichloroacetic acid (TCA) solution and the quantity of soluble protein remaining in it. Neutral detergent insoluble nitrogen (NDIN) and acid detergent insoluble nitrogen (ADIN) were estimated by nitrogen dosing in the NDF and NDA residues, respectively, according to the method described by Licitra et al. (1996).

The carbohydrate fractions were determined according to the protocol in Sniffen et al. (1992).

In vitro incubations were conducted in $100-\mathrm{mL}$ amber flasks containing 0.50-g incubated sample, 40 $\mathrm{mL}$ culture medium, and $10-\mathrm{mL}$ ruminal inoculum sealed with rubber stoppers and aluminum foil. The ruminal inoculum was obtained from three rumenfistulated sheep fed once daily with corn silage and soybean meal in the proportion of nine parts forage to

Pesq. agropec. bras., Brasília, v.52, n.12, p.1268-1275, dez. 2017 DOI: 10.1590/S0100-204X2017001200016 
one part concentrate. The culture medium was prepared according to Hall \& Mertens (2008). It consisted of 40-mL reduced solution and $10-\mathrm{mL}$ ruminal inoculum under $\mathrm{CO}_{2}$ aspersion. The flasks were sealed and kept in a water bath maintained at $39^{\circ} \mathrm{C}$.

The time profiles of accumulated gas production were obtained using a nonautomated device similar to that described by Abreu et al. (2014). The gas pressure was determined from manometric readings, and the volume was measured with a graduated pipette $(25 \mathrm{~mL}$ with $0.1 \mathrm{~mL}$ gradations). For these determinations, a manometer ( $0-8$ psi; 0.05 psi gradations) was coupled to a three-way plastic valve in a wood frame. Pressure and volume measurements were performed on each bottle at $0,2,4,6,8,10,12,16,20,30,36,48,72$, and 96 hours. The volume at each time interval and the total gas volume were used in a model to estimate kinetic parameters.

Gas production data were analyzed using a the biphasic model, composed by the monomolecular and GNG1 models (Vieira et al., 2008), according to the equation:

$$
\begin{aligned}
V_{t} & =V_{f 1} \times\left(1-\exp \left(-k_{1} t\right)+V_{f 2}\right) \times 1- \\
& \left(\delta^{N} \exp \left(-k_{2} t\right)+\exp (-\lambda t) \sum_{i=1}^{N-1} \frac{\left(1-\delta^{N-i}\right)(\lambda t)^{i}}{i !}\right),
\end{aligned}
$$

in which $V_{t}$ is the final volume of gases accumulated $(\mathrm{mL})$ in time $(\mathrm{t}) ; \mathrm{V}_{\mathrm{fl}}$ is the gas volume from the soluble fraction $(\mathrm{mL}) ; \mathrm{k}_{1}$ is the fermentation rate of the soluble fraction $\left(\% \mathrm{~h}^{-1}\right) ; \mathrm{V}_{\mathrm{f} 2}$ is the gas volume from the fibrous

Table 1. Chemical composition of elephant grass (Pennisetum purpureum) hay harvested after flowering.

\begin{tabular}{lc}
\hline Component & Mean $(95 \% \mathrm{CI})$ \\
\hline Dry matter $\left(\mathrm{g} \mathrm{kg}^{-1}\right)$ & $893.7 \pm 6.01$ \\
$\mathrm{Ash}\left(\mathrm{g} \mathrm{kg}^{-1} \mathrm{DM}\right)$ & $83.2 \pm 1.61$ \\
Ether extract $\left(\mathrm{g} \mathrm{kg}^{-1} \mathrm{DM}\right)$ & $14.6 \pm 0.47$ \\
Neutral detergent fiber $\left(\mathrm{g} \mathrm{kg}^{-1} \mathrm{DM}\right)$ & $758.7 \pm 5.14$ \\
Acid detergent fiber $\left(\mathrm{g} \mathrm{kg}^{-1} \mathrm{DM}\right)$ & $470.6 \pm 3.91$ \\
Hemicellulose $\left(\mathrm{g} \mathrm{kg}^{-1} \mathrm{DM}\right)$ & $347.6 \pm 5.50$ \\
Cellulose $\left(\mathrm{g} \mathrm{kg}^{-1} \mathrm{DM}\right)$ & $288.5 \pm 3.18$ \\
Lignin $\left(\mathrm{g} \mathrm{kg}^{-1} \mathrm{DM}\right)$ & $91.1 \pm 2.55$ \\
Crude protein $(\mathrm{g} \mathrm{kg}$ & $\mathrm{DM})$ \\
Neutral detergent insoluble nitrogen $\left(\mathrm{g} \mathrm{kg}^{-1} \mathrm{~N}\right)$ & $485.6 \pm 1.07$ \\
Acid detergent insoluble nitrogen $\left(\mathrm{g} \mathrm{kg}^{-1} \mathrm{~N}\right)$ & $212.3 \pm 17.28$ \\
\hline (1) $\mathrm{CI}$ confidence interval. & $108.0 \pm 9.22$ \\
\hline
\end{tabular}

${ }^{(1)} \mathrm{CI}$, confidence interval. fraction ( $\mathrm{mL}) ; \mathrm{k}_{2}$ is the fermentation rate in the fibrous fraction $\left(\% \mathrm{~h}^{-1}\right) ; \mathrm{N}$ is a positive whole number indicating the order of time dependency; $\lambda$ is the asymptote of the rate of preparation for digestion $\left(\% \mathrm{~h}^{-1}\right) ; \delta^{\mathrm{N}}$ is a constant; and $i$ is a subscript denoting the order of time dependency ranging from 1 to $\mathrm{Na}$ or $\mathrm{Nb}$. This model presented the best fit according to the Akaike criterion, and was determined using the NLMIXED module of SAS (SAS Institute, Inc, Cary, NC, USA).

The data were subjected to the analysis of variance, and the means for urease sources and their levels were analyzed by Tukey's test and the regression analysis, respectively, at 5\% probability, using the ExpDes.pt package from the Rstudio software (Ferreira et al., 2013).

\section{Results and Discussion}

There were no significant differences between the control and the interaction of the factorial arrangements for dry matter, ash, and ether extract. However, an effect of urease source on ether extract content was observed (Table 3). The hay treated with soybean had a higher ether extract content than that treated with jack bean or pigeon pea because soybean itself yielded a higher ether extract content than either jack bean or pigeon pea (Table 2). Ahmed et al. (2002) reported that the ether extract content in roughage ammoniated with urea and treated with $6 \%$ soybean was $18.5 \mathrm{~g} \mathrm{~kg}^{-1}$ higher than that of the control.

For the NDF content, significant differences were observed between the control and the treatment

Table 2. Chemical composition of milled soybean (Glycine max), pigeon pea (Cajanus cajan), and jack bean (Canavalia ensiformis) grains used as urease sources.

\begin{tabular}{lccc}
\hline Components & \multicolumn{3}{c}{ Urease source $(95 \% \mathrm{CI})$} \\
\cline { 2 - 4 } & Jack bean & Pigeon pea & Soybean \\
\hline Dry matter $\left(\mathrm{g} \mathrm{kg}^{-1}\right)$ & $884.3 \pm 8.27$ & $879.2 \pm 9.27$ & $902.6 \pm 7.27$ \\
Ash $\left(\mathrm{g} \mathrm{kg}^{-1} \mathrm{DM}\right)$ & $32.5 \pm 2.27$ & $39.4 \pm 2.36$ & $48.7 \pm 1.89$ \\
Ether extract $\left(\mathrm{g} \mathrm{kg}^{-1} \mathrm{DM}\right)$ & $21.1 \pm 0.95$ & $19.6 \pm 0.81$ & $28.3 \pm 0.73$ \\
Crude protein $\left(\mathrm{g} \mathrm{kg}^{-1} \mathrm{DM}\right)$ & $316.5 \pm 2.79$ & $206.6 \pm 1.23$ & $334.5 \pm 1.73$ \\
$\mathrm{NDF}\left(\mathrm{g} \mathrm{kg}^{-1} \mathrm{DM}\right)$ & $255.9 \pm 5.79$ & $375.3 \pm 6.23$ & $296.8 \pm 4.23$ \\
$\mathrm{ADF}\left(\mathrm{g} \mathrm{kg}^{-1} \mathrm{DM}\right)$ & $122.3 \pm 3.92$ & $174.6 \pm 3.79$ & $126.9 \pm 4.23$ \\
Hemicellulose $\left(\mathrm{g} \mathrm{kg}^{-1} \mathrm{DM}\right)$ & $133.6 \pm 2.38$ & $200.7 \pm 3.14$ & $169.9 \pm 2.68$ \\
Urea activity index $(\Delta \mathrm{pH})$ & $1.78 \pm 0.20$ & $2.21 \pm 0.13$ & $2.76 \pm 0.18$ \\
\hline
\end{tabular}

$\mathrm{CI}$, confidence interval, NDF, neutral detergent fiber; $\mathrm{ADF}$, acid detergent fiber. 
combinations. However, urease source level had no effect (Table 4). A significant interaction was observed between the factors analyzed for NDF, ADF, and lignin content. The addition of $3 \%$ jack bean as a source of urease increased NDF more than the same amount of soybean or pigeon pea. The addition of 1

Table 3. Chemical composition of elephant grass (Pennisetum purpureum) hay ammoniated with urea, using soybean (Glycine max), jack bean (Canavalia ensiformis), or pigeon pea (Cajanus cajan) as a urease source ${ }^{(1)}$.

\begin{tabular}{|c|c|c|c|c|c|c|c|c|c|c|c|c|}
\hline \multirow{2}{*}{$\begin{array}{l}\text { Urease } \\
\text { source }\end{array}$} & \multirow[t]{2}{*}{ Control } & \multirow{2}{*}{$\begin{array}{l}\text { Mean } \\
\text { (US) }\end{array}$} & \multicolumn{4}{|c|}{ Level (\% DM) } & \multirow{2}{*}{$\begin{array}{c}\text { Factorial } \\
\text { mean }\end{array}$} & \multirow{2}{*}{$\begin{array}{l}\mathrm{CV} \\
(\%)\end{array}$} & \multicolumn{4}{|c|}{$\mathrm{p}$-value } \\
\hline & & & 1 & 2 & 3 & 4 & & & $\mathrm{~L}$ & US & USxL & FAxC \\
\hline & \multicolumn{12}{|c|}{ Dry matter $\left(\mathrm{DM}, \mathrm{g} \mathrm{kg}^{-1}\right)$} \\
\hline Soybean & & 742.0 & 740.9 & 738.5 & 753.6 & 735.1 & & & & & & \\
\hline Jack bean & 742.4 & 736.8 & 739.0 & 739.0 & 736.0 & 733.2 & 741.9 & 1.52 & ns & ns & ns & ns \\
\hline \multirow[t]{2}{*}{ Pigeon pea } & & 743.6 & 750.8 & 745.9 & 740.2 & 737.3 & & & & & & \\
\hline & \multicolumn{12}{|c|}{ Ash $\left(\mathrm{g} \mathrm{kg}^{-1} \mathrm{DM}\right)$} \\
\hline Soybean & & 84.3 & 85.4 & 83.1 & 81.6 & 87.5 & & & & & & \\
\hline Jack bean & 84.9 & 81.7 & 82.6 & 80.8 & 81.4 & 82.1 & 83.7 & 4.88 & ns & ns & ns & $\mathrm{ns}$ \\
\hline \multirow[t]{2}{*}{ Pigeon pea } & & 85.1 & 85.8 & 84.3 & 84.6 & 86.0 & & & & & & \\
\hline & \multicolumn{12}{|c|}{ Ether extract $\left(\mathrm{g} \mathrm{kg}^{-1} \mathrm{DM}\right)$} \\
\hline Soybean & & $16.1 \mathrm{a}$ & 16.1 & 16.4 & 16.0 & 16.1 & & & & & & \\
\hline Jack bean & 14.0 & $13.8 \mathrm{~b}$ & 13.6 & 14.0 & 13.1 & 14.6 & 14.59 & 11.56 & ns & ** & ns & ns \\
\hline Pigeon pea & & $13.8 \mathrm{~b}$ & 13.1 & 15.1 & 12.8 & 14.4 & & & & & & \\
\hline
\end{tabular}

${ }^{n}$ Nonsignificant. **Significant by Fisher's test, at $1 \%$ probability. US x L, interaction between urease source and level and FA x C, factorial and control difference.

Table 4. Fibrous fraction components $\left(\mathrm{g} \mathrm{kg}^{-1} \mathrm{DM}\right)$ of elephant grass (Pennisetum purpureum) hay ammoniated with urea, using milled soybean (Glycine max), jack bean (Canavalia ensiformis), or pigeon pea (Cajanus cajan) as a urease source ${ }^{(1)}$.

\begin{tabular}{|c|c|c|c|c|c|c|c|c|c|c|c|c|}
\hline \multirow{2}{*}{$\begin{array}{l}\text { Urease } \\
\text { source }\end{array}$} & \multirow{2}{*}{ Control } & \multirow{2}{*}{$\begin{array}{c}\text { Mean } \\
\text { (US) }\end{array}$} & \multicolumn{4}{|c|}{ Level (\% DM) } & \multirow{2}{*}{$\begin{array}{c}\text { Factorial } \\
\text { mean }\end{array}$} & \multirow{2}{*}{$\begin{array}{l}\text { CV } \\
(\%)\end{array}$} & \multicolumn{4}{|c|}{$\mathrm{p}$-value } \\
\hline & & & 1 & 2 & 3 & 4 & & & $\mathrm{~L}$ & US & US x L & FA $x \mathrm{C}$ \\
\hline & \multicolumn{12}{|c|}{ Neutral detergent fiber } \\
\hline Soybean & \multirow{3}{*}{$749.2 \mathrm{~A}$} & 740.4 & $744.4 \mathrm{a}$ & $741.6 \mathrm{a}$ & $736.5 b$ & $739.1 \mathrm{a}$ & \multirow{3}{*}{$740.8 \mathrm{~B}$} & \multirow{3}{*}{0.93} & \multirow{3}{*}{ ns } & \multirow{3}{*}{ ns } & \multirow{3}{*}{$*$} & \multirow{3}{*}{$* *$} \\
\hline Jack bean & & 741.9 & $744.5 \mathrm{a}$ & $737.4 \mathrm{a}$ & $751.5 \mathrm{a}$ & $734.2 \mathrm{a}$ & & & & & & \\
\hline \multirow[t]{2}{*}{ Pigeon pea } & & 741.2 & $742.5 \mathrm{a}$ & $742.5 \mathrm{a}$ & $737.4 b$ & $742.3 \mathrm{a}$ & & & & & & \\
\hline & \multicolumn{12}{|c|}{ Acid detergent fiber } \\
\hline Soybean & \multirow{3}{*}{448.0} & 453.3 & $457.0 \mathrm{a}$ & $455.3 \mathrm{a}$ & $452.3 \mathrm{a}$ & $449.2 \mathrm{a}$ & \multirow{3}{*}{450.5} & \multirow{3}{*}{1.84} & \multirow{3}{*}{ ns } & \multirow{3}{*}{ ns } & \multirow{3}{*}{$*$} & \multirow{3}{*}{ ns } \\
\hline Jack bean & & 446.5 & $442.7 b$ & $441.0 \mathrm{~b}$ & $456.1 \mathrm{a}$ & $446.4 \mathrm{a}$ & & & & & & \\
\hline \multirow[t]{2}{*}{ Pigeon pea } & & 451.8 & $446.8 \mathrm{ab}$ & $456.5 \mathrm{a}$ & $446.7 \mathrm{a}$ & $455.9 \mathrm{a}$ & & & & & & \\
\hline & \multicolumn{12}{|c|}{ Hemicellulose } \\
\hline Soybean & \multirow{3}{*}{301.1} & 289.6 & 287.3 & 289.4 & 292.4 & 289.2 & \multirow{3}{*}{293.4} & \multirow{3}{*}{3.44} & \multirow{3}{*}{ ns } & \multirow{3}{*}{ ns } & \multirow{3}{*}{ ns } & \\
\hline Jack bean & & 297.1 & 304.4 & 298.5 & 296.3 & 289.3 & & & & & & ns \\
\hline Pigeon pea & & 293.0 & 299.3 & 290.2 & 293.3 & 289.2 & & & & & & \\
\hline & & & & & & Cellulos & & & & & & \\
\hline Soybean & & 340.0 & 341.4 & 338.6 & 346.9 & 333.2 & & & & & & \\
\hline Jack bean & 331.9 & 333.7 & 331.3 & 331.5 & 340.8 & 331.3 & 336.3 & 2.25 & ns & ns & ns & ns \\
\hline Pigeon pea & & 335.3 & 330.7 & 338.4 & 332.7 & 339.2 & & & & & & \\
\hline & & & & & & Lignin & & & & & & \\
\hline Soybean & & 79.2 & $81.8 \mathrm{a}$ & $82.3 \mathrm{a}$ & $72.4 \mathrm{~b}$ & $80.3 a$ & & & & & & \\
\hline Jack bean & 84.4 & 78.6 & $77.3 \mathrm{a}$ & $75.8 b$ & $82.0 \mathrm{a}$ & $79.4 \mathrm{a}$ & 80.1 & 5.61 & ns & ns & $*$ & ns \\
\hline Pigeon pea & & 82.5 & $84.4 \mathrm{a}$ & $83.1 \mathrm{a}$ & $81.0 \mathrm{a}$ & $81.5 \mathrm{a}$ & & & & & & \\
\hline
\end{tabular}


and $2 \%$ jack bean resulted in the lowest ADF content, whereas adding $2 \%$ jack bean or $3 \%$ soybean yielded the lowest lignin value. These findings corroborate those reported by Khan et al. (1999), who added milled jack bean and soybean to ammoniated wheat straw and found that a 1:1 ratio of milled legume seeds to urea reduced fiber content the most. When Sarmento et al. (2001) ammoniated sugarcane (Saccharum officinarum) bagasse with $4 \%$ urea and added ground raw soybean as a urease source, they observed a reduction of $2.9 \%$ in NDF content, compared with the control, with the addition of $7.5 \%$ soybean. These results confirmed the hypothesis that adding a urease source at the time of ammonization accelerates urea hydrolysis and ammonia liberation (Khan et al., 1999).
There were significant differences between the control and the combination of factorial arrangements for the $\mathrm{A}+\mathrm{B} 1$ fraction. However, neither the urease source nor its concentration had any significant effect on the carbohydrate fractions (Table 5).

The increase in the $\mathrm{A}+\mathrm{B} 1$ fraction (nonfibrous carbohydrate) content indicates a rise in rapidly degradable soluble compounds, such as sugars, and a reduction in the fibrous carbohydrate content. The urease source only had a significant effect on the nitrogenous compounds NPN and ADIN (Table 6). Regardless of its concentration, jack bean lowered ADIN more than soybean or pigeon pea. Soybean increased NPN the most, so its urease activity is higher than that of the others legumes evaluated in the present study (Table 2). Therefore, as a urease source,

Table 5. Carbohydrate fractions of elephant grass (Pennisetum purpureum) hay ammoniated with urea, using milled soybean (Glycine max), pigeon pea (Cajanus cajan), or jack bean (Canavalia ensiformis) as a urease source ${ }^{(1)}$.

\begin{tabular}{|c|c|c|c|c|c|c|c|c|c|c|c|c|}
\hline \multirow{2}{*}{$\begin{array}{l}\text { Urease } \\
\text { source }\end{array}$} & \multirow[t]{2}{*}{ Control } & \multirow{2}{*}{$\begin{array}{l}\text { Mean } \\
\text { (US) }\end{array}$} & \multicolumn{4}{|c|}{ Level (\% DM) } & \multirow{2}{*}{$\begin{array}{c}\text { Factorial } \\
\text { mean }\end{array}$} & \multirow{2}{*}{$\begin{array}{l}\mathrm{CV} \\
(\%)\end{array}$} & \multicolumn{4}{|c|}{ p-value } \\
\hline & & & 1 & 2 & 3 & 4 & & & $\mathrm{~L}$ & US & US x L & FA $x \mathrm{C}$ \\
\hline & \multicolumn{12}{|c|}{ Total carbohydrate $\left(\mathrm{g} \mathrm{kg}^{-1} \mathrm{DM}\right)$} \\
\hline Soybean & & 785.6 & 784.8 & 786.8 & 794.2 & 776.7 & & & & & & \\
\hline Jack bean & 781.0 & 786.0 & 792.0 & 796.5 & 793.2 & 790.3 & 788.2 & 1.47 & ns & $\mathrm{ns}$ & $\mathrm{ns}$ & ns \\
\hline \multirow[t]{2}{*}{ Pigeon pea } & & 785.6 & 786.8 & 787.7 & 788.4 & 781.6 & & & & & & \\
\hline & \multicolumn{12}{|c|}{ Neutral detergent fiber corrected for ash and protein content $\left(\mathrm{g} \mathrm{kg}^{-1} \mathrm{TC}\right)$} \\
\hline Soybean & & 688.7 & 686.0 & 686.9 & 694.1 & 697.5 & & & & & & \\
\hline Jack bean & 691.9 & 686.5 & 689.9 & 683.5 & 695.7 & 677.2 & 687.4 & 1.56 & ns & ns & $\mathrm{ns}$ & $\mathrm{ns}$ \\
\hline \multirow[t]{2}{*}{ Pigeon pea } & & 687.0 & 685.2 & 684.9 & 688.5 & 689.5 & & & & & & \\
\hline & \multicolumn{12}{|c|}{ Nonfibrous carbohydrates $\left(\mathrm{g} \mathrm{kg}^{-1} \mathrm{TC}\right)$} \\
\hline Soybean & & 95.2 & 88.7 & 105.9 & 109.2 & 88.8 & & & & & & \\
\hline Jack bean & $93.6 \mathrm{~B}$ & 105.3 & 99.3 & 111.7 & 97.5 & 113.1 & $100.7 \mathrm{~A}$ & 13.02 & $\mathrm{~ns}$ & $\mathrm{~ns}$ & $\mathrm{~ns}$ & $* *$ \\
\hline \multirow[t]{2}{*}{ Pigeon pea } & & 101.6 & 96.6 & 102.7 & 109.9 & 97.5 & & & & & & \\
\hline & \multicolumn{12}{|c|}{ Potentially biodegradable neutral detergent fiber $\left(\mathrm{g} \mathrm{kg}^{-1} \mathrm{TC}\right)$} \\
\hline Soybean & & 500.5 & 495.8 & 494.8 & 522.5 & 499.8 & & & & & & \\
\hline Jack bean & 502.9 & 497.6 & 507.1 & 503.7 & 493.6 & 486.4 & 495.3 & 3.7 & ns & ns & $\mathrm{ns}$ & ns \\
\hline \multirow[t]{2}{*}{ Pigeon pea } & & 487.7 & 487.6 & 480.6 & 495.1 & 487.8 & & & & & & \\
\hline & \multicolumn{12}{|c|}{ Nondegradable fraction $\left(\mathrm{g} \mathrm{kg}^{-1} \mathrm{TC}\right)$} \\
\hline Soybean & & 192.5 & 200.3 & 197.1 & 174.2 & 198.7 & & & & & & \\
\hline Jack bean & 184.5 & 190.1 & 185.6 & 182.0 & 202.1 & 190.8 & 194.7 & 8.25 & $\mathrm{~ns}$ & $\mathrm{~ns}$ & $\mathrm{~ns}$ & $\mathrm{~ns}$ \\
\hline Pigeon pea & & 201.5 & 202.6 & 204.3 & 194.5 & 204.9 & & & & & & \\
\hline
\end{tabular}

${ }^{(1)}$ Means followed by different letters between control and factorial mean differ significantly by Tukey's test at 5\% probability. L, urease level; US, urease source; US x L, interaction between urease source and level; and FA x C, factorial and control difference. DM, dry matter; and CV, coefficient of

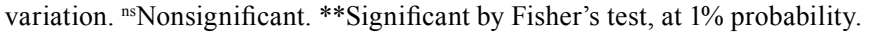


soybean can be applied in smaller amounts than jack bean or pigeon pea. Reis et al. (2001) reported increases in the NPN/TN content of ammoniated Jaragua grass (Hyparrhenia rufa) hay when lablab (Lablab purpureus) was used as the urease source. Pereira et al. (2010) highlighted that the increase in NPN content indicates that higher amounts of nitrogen are available for the ruminal bacteria that degrade fibrous carbohydrates, due to their capacity of assimilating this form of nitrogen.

There was a significant interaction between urease source and application rate for the gas volume generated from nonfibrous carbohydrates $\left(\mathrm{V}_{\mathrm{fl}}\right)$. However, there was no significant interaction between these factors for the fermentation rate of nonfibrous carbohydrates $\left(\mathrm{k}_{1}\right)$, latency $(\mathrm{L})$, gas volume produced by fibrous carbohydrates $\left(\mathrm{Vf}_{2}\right)$, or fermentation rate of fibrous carbohydrates $\left(\mathrm{k}_{2}\right)$. A positive linear correlation was observed for $\mathrm{Vf}_{1}$ when soybean was used as the urease source (Table 7). The increase in gas production from the fibrous fraction can be attributed to the addition of non protein nitrogen and soluble carbohydrates to the roughage (Velásquez et al., 2010). It is also the result of the improvement of fiber quality, as the ammonia-promoted breakage of cellulose and lignin bonds improves the access of ruminal microorganisms to the fiber and increases gas production (Abo-Donia et al., 2014).

Sarmento et al. (2001) treated ammoniated sugarcane bagasse with $4 \%$ urea then added $0,2.5$, 3.75 , or $7.5 \%$ ground soybean to it as a urease source. These authors reported a quadratic response of in vitro dry matter digestibility as a function of soybean content and concluded that $3.75 \%$ ground soybean improved the digestibility of the dry matter in sugarcane bagasse. Ahmed et al. (2002) ammoniated rice (Oryza sativa) straw with $4 \%$ urea, adding 0 , 4 , or $6 \%$ ground soybean, and found that the total digestibility coefficient significantly increased, compared with the control treatment, when up to $6 \%$ milled legume seeds were added to the ruminant diet.

Table 6. Nitrogenous fractions of elephant grass (Pennisetum purpureum) hay ammoniated with urea and to which milled soybean (Glycine max), pigeon pea (Cajanus cajan), or jack bean (Canavalia ensiformis) was added as a urease source ${ }^{(1)}$.

\begin{tabular}{|c|c|c|c|c|c|c|c|c|c|c|c|c|}
\hline \multirow{2}{*}{$\begin{array}{l}\text { Urease } \\
\text { source }\end{array}$} & \multirow[t]{2}{*}{ Control } & \multirow{2}{*}{$\begin{array}{c}\text { Mean } \\
\text { (US) }\end{array}$} & \multicolumn{4}{|c|}{ Level (\% DM) } & \multirow{2}{*}{$\begin{array}{c}\text { Factorial } \\
\text { mean }\end{array}$} & \multirow{2}{*}{$\begin{array}{l}\mathrm{CV} \\
(\%)\end{array}$} & \multicolumn{4}{|c|}{$\mathrm{p}$ - value } \\
\hline & & & 1 & 2 & 3 & 4 & & & L & US & US $\times \mathrm{L}$ & FA $\times \mathrm{C}$ \\
\hline & \multicolumn{12}{|c|}{ Total nitrogen (g kg-1 DM) } \\
\hline Soybean & & 18.2 & 18.2 & 18.2 & 17.3 & 19.2 & & & & & & \\
\hline Jack bean & 18.1 & 17.9 & 17.9 & 17.4 & 18.0 & 18.1 & 18.7 & 6.15 & ns & $\mathrm{ns}$ & $\mathrm{ns}$ & ns \\
\hline \multirow[t]{2}{*}{ Pigeon pea } & & 18.4 & 18.3 & 18.1 & 18.3 & 18.9 & & & & & & \\
\hline & \multicolumn{12}{|c|}{ Neutral detergent insoluble nitrogen ( $\mathrm{g} \mathrm{kg}^{-1}$ total nitrogen) } \\
\hline Soybean & & 255.4 & 254.8 & 251.3 & 264.2 & 251.2 & & & & & & \\
\hline Jack bean & 248.2 & 270.3 & 279.8 & 256.5 & 271.3 & 273.4 & 264.1 & 8.88 & ns & $\mathrm{ns}$ & $\mathrm{ns}$ & ns \\
\hline \multirow[t]{2}{*}{ Pigeon pea } & & 266.6 & 268.2 & 299.1 & 254.6 & 244.5 & & & & & & \\
\hline & \multicolumn{12}{|c|}{ Acid detergent insoluble nitrogen $\left(\mathrm{g} \mathrm{kg}^{-1}\right.$ total nitrogen $)$} \\
\hline Soybean & & $111.5 b$ & 108.4 & 106.8 & 108.7 & 122.2 & & & & & & \\
\hline Jack bean & 84.5 & $99.3 \mathrm{a}$ & 94.0 & 105.4 & 104.8 & 93.1 & 107.7 & 9.32 & ns & $* *$ & ns & ns \\
\hline \multirow[t]{2}{*}{ Pigeon pea } & & $112.2 \mathrm{~b}$ & 101.9 & 112.0 & 121.5 & 113.3 & & & & & & \\
\hline & \multicolumn{12}{|c|}{ Non protein nitrogen $\left(\mathrm{g} \mathrm{kg}^{-1}\right.$ total nitrogen $)$} \\
\hline Soybean & & $493.3 \mathrm{a}$ & 520.0 & 471.4 & 485.2 & 496.6 & & & & & & \\
\hline Jack bean & 436.9B & $455.4 \mathrm{~b}$ & 479.1 & 448.2 & 432.4 & 461.9 & $461.1 \mathrm{~A}$ & 8.99 & ns & $*$ & ns & $* *$ \\
\hline Pigeon pea & & $434.5 b$ & 468.4 & 421.1 & 440.1 & 408.3 & & & & & & \\
\hline
\end{tabular}


Table 7. In vitro gas production kinetic parameters (P) of elephant grass (Pennisetum purpureum) hay treated with urea, using milled soybean (Glycine max), pigeon pea (Cajanus cajan), or jack bean (Canavalia ensiformis) as a urease source ${ }^{(1)}$.

\begin{tabular}{|c|c|c|c|c|c|c|c|c|c|c|c|}
\hline \multirow{2}{*}{$\begin{array}{l}\text { Urease } \\
\text { source }\end{array}$} & \multirow[t]{2}{*}{ Control } & \multirow{2}{*}{$\begin{array}{c}\text { Mean } \\
\text { (US) }\end{array}$} & \multicolumn{4}{|c|}{ Level (\% DM) } & \multirow{2}{*}{$\begin{array}{c}\text { Factorial } \\
\text { mean }\end{array}$} & \multirow{2}{*}{$\begin{array}{l}\text { CV } \\
(\%)\end{array}$} & \multirow{2}{*}{\multicolumn{2}{|c|}{ Regression }} & \multirow[t]{2}{*}{$\mathrm{R}^{2}$} \\
\hline & & & 1 & 2 & 3 & 4 & & & & & \\
\hline & \multicolumn{11}{|c|}{ Gas volume from soluble fraction $\left(\mathrm{V}_{\mathrm{fl}}, \mathrm{mL}\right)$} \\
\hline Soybean & \multirow{3}{*}{$8.5 \mathrm{~B}$} & $9.0 \mathrm{a}$ & 8.2 & 8.2 & 8.4 & 11.2 & & & \multicolumn{2}{|c|}{$* * \hat{\mathrm{Y}}=6.72+0.92 \mathrm{x}$} & 0.67 \\
\hline Jack bean & & $8.5 b$ & 8.3 & 8.6 & 8.3 & 8.6 & $8.9 \mathrm{~A}$ & 7.13 & ns & - & - \\
\hline \multirow[t]{2}{*}{ Pigeon pea } & & $8.1 \mathrm{~b}$ & 8.3 & 7.8 & 7.8 & 8.4 & & & ns & - & - \\
\hline & \multicolumn{11}{|c|}{ Fermentation rate of soluble fraction $\left(\mathrm{k}_{1}, \% \mathrm{~h}^{-1}\right)$} \\
\hline Soybean & \multirow{3}{*}{0.18} & 0.20 & 0.20 & 0.20 & 0.21 & 0.20 & & & ns & - & - \\
\hline Jack bean & & 0.20 & 0.19 & 0.20 & 0.20 & 0.19 & 0.19 & 6.11 & ns & - & - \\
\hline \multirow[t]{2}{*}{ Pigeon pea } & & 0.20 & 0.19 & 0.19 & 0.2 & 0.20 & & & ns & - & - \\
\hline & \multicolumn{11}{|c|}{ Latency $(\mathrm{L}, \mathrm{h})$} \\
\hline Soybean & & 1.55 & 1.46 & 1.66 & 1.6 & 1.46 & & & ns & - & - \\
\hline Jack bean & 1.49 & 1.44 & 1.44 & 1.41 & 1.5 & 1.39 & 1.6 & 27.2 & ns & - & - \\
\hline \multirow[t]{2}{*}{ Pigeon pea } & & 1.51 & 1.43 & 1.38 & 1.62 & 1.59 & & & ns & - & - \\
\hline & \multicolumn{11}{|c|}{ Gas volume from fibrous fraction $\left(\mathrm{V}_{\mathrm{f} 2}, \mathrm{~mL}\right)$} \\
\hline Soybean & & 17.0 & 17.6 & 16.6 & 17 & 16.6 & & & ns & - & - \\
\hline Jack bean & 16.0 & 16.6 & 16.4 & 16.5 & 16.6 & 16.7 & 16.8 & 4.61 & ns & - & - \\
\hline \multirow[t]{2}{*}{ Pigeon pea } & & 16.9 & 16.9 & 16.4 & 16.4 & 17.9 & & & ns & - & - \\
\hline & \multicolumn{11}{|c|}{ Fermentation rate of fibrous fraction $\left(\mathrm{k}_{2}, \% \mathrm{~h}^{-1}\right)$} \\
\hline Soybean & & 0.015 & 0.013 & 0.016 & 0.016 & 0.016 & & & ns & - & - \\
\hline Jack bean & 0.016 & 0.016 & 0.016 & 0.016 & 0.016 & 0.015 & 0.014 & 14.61 & $\mathrm{~ns}$ & - & - \\
\hline Pigeon pea & & 0.014 & 0.015 & 0.014 & 0.015 & 0.013 & & & ns & - & - \\
\hline
\end{tabular}

\section{Conclusion}

Adding milled soybean (Glycine max), jack bean (Canavalia ensiformis), and pigeon pea (Cajanus cajan) as urease sources to elephant grass (Pennisetum purpureum) hay ammoniated with urea contributes to the reduction of neutral detergent fiber and acid detergent insoluble nitrogen, and promotes an increase in nonfibrous carbohydrates and non protein nitrogen content for elephant grass hay ammoniated with urea.

\section{Acknowledgments}

To Coordenação de Aperfeiçoamento de Pessoal de Nível Superior (Capes) and to Conselho Nacional de Desenvolvimento Científico e Tecnológico (CNPq), for scholarships granted.

\section{References}

ABO-DONIA, F.M.; ABDEL-AZIM, S.N.; ELGHANDOUR, M.M.Y.; SALEM, A.Z.M.; BUENDÍA, G.; SOLIMAN, N.A.M. Feed intake, nutrient digestibility and ruminal fermentation activities in sheep-fed peanut hulls treated with Trichoderma viride or urea. Tropical Animal Health and Production, v.46, p.221-228, 2014. DOI: 10.1007/s11250-013-0479-z.

ABREU, M.L.C.; VIEIRA, R.A.M.; ROCHA, N.S.; ARAÚJO, R.P.; GLÓRIA, L.S.; FERNANDES, A.M.; LACERDA, P.D. de; GESUALDI JÚNIOR, A. Clitoria ternatea L, as a potential high quality forage legume. Asian-Australasian Journal of Animal Sciences, v.27, p.169-178, 2014. DOI: 10.5713/ajas.2013.13343.

AHMED, S.; KHAN, M.J.; SHAHJALAL, M.; ISLAM, K.M.S. Effects of feeding urea and soybean meal-treated rice straw on digestibility of feed nutrients and growth performance of bull calves. Asian-Australasian Journal of Animal Sciences, v.15, p.522-527, 2002. DOI: 10.5713/ajas.2002.522.

BALASUBRAMANIAN, A.; DURAIRAJPANDIAN, V.; ELUMALAI, S.; MATHIVANAN, N.; MUNIRAJAN, A.K.; PONNURAJ, K. Structural and functional studies on urease from pigeon pea (Cajanus cajan). International Journal of Biological Macromolecules, v.58, p.301-309, 2013. DOI: 10.1016/j. ijbiomac.2013.04.055.

BERTIPAGLIA, L.M.A.; DE LUCA, S.; MELO, G.M.P. de; REIS, R.A. Avaliação de fontes de urease na amonização de fenos de Brachiaria brizantha com dois teores de umidade. Revista Brasileira de Zootecnia, v.34, p.378-386, 2005. DOI: 10.1590/ S1516-35982005000200004.

CARVALHO, G.G.P. de; PIRES, A.J.V.; GARCIA, R.; SILVA, R.R.; MENDES, F.B.L.; PINHEIRO, A.A.; SOUZA, D.R. de. Degradabilidade in situ da matéria seca e da fração fibrosa do 
bagaço de cana-de-açúcar tratado com uréia. Ciência Animal Brasileira, v.8, p.447-455, 2007.

FERREIRA, E.B.; CAVALCANTI, P.P.; NOGUEIRA, D.A. ExpDes: Experimental Designs package. 2013. R package version 1.1.2. Available at: <http://CRAN.R-project.org/ package $=$ ExpDes $>$. Accessed on: Mar. 72016 .

GOMES, J.C.; OLIVEIRA, G.F. Análises físico-químicas de alimentos. Viçosa: Ed. da UFV, 2011. 303p.

HALL, M.B.; MERTENS, D.R. In vitro fermentation vessel type and method alter fiber digestibility estimates. Journal of Dairy Science, v.91, p.301-307, 2008. DOI: 10.3168/jds.2006-689.

HELRICH, K. (Ed.). Official methods of analysis of the AOAC. $15^{\text {th }}$ ed. Arlington: Association of Official Analytical chemists, 1990. 1094p.

KHAN, M.J.; SCAIFE, J.R.; HOVELL, F.D. The effect of different sources of urease enzyme on the nutritive value of wheat straw treated with urea as a source of ammonia. Asian-Australasian Journal of Animal Sciences, v.12, p.1063-1069, 1999. DOI: 10.5713/ajas.1999.1063.

LICITRA, G.; HERNANDEZ, T.M.; VAN SOEST, P.J. Standardization of procedures for nitrogen fractionation of ruminant feeds. Animal Feed Science and Technology, v.57, p.347-358, 1996. DOI: 10.1016/0377-8401(95)00837-3.

MEDEIROS-SILVA, M.; FRANCK, W.L.; BORBA, M.P.; PIZZATO, S.B.; STRODTMAN, K.N.; EMERICH, D.W.; STACEY, G.; POLACCO, J.C.; CARLINI, C.R. Soybean ureases, but not that of Bradyrhizobium japonicum, are involved in the process of soybean root nodulation. Journal of Agricultural and Food Chemistry, v.62, p.3517-3524, 2014. DOI: 10.1021/ jf5000612.

PEREIRA, E.S.; PIMENTEL, P.G.; DUARTE, L.S.; MIZUBUTI, I.Y.; ARAUJO, G.G.L. de; CARNEIRO, M.S. de S.; REGADAS FILHO, J.G.L.; MAIA, I.S.G. Determinação das frações proteicas e de carboidratos e estimativa do valor energético de forrageiras e subprodutos da agroindústria produzidos no Nordeste Brasileiro. Semina: Ciências Agrárias, v.31, p.1079-1094, 2010. DOI: 10.5433/1679-0359.2010v31n4p1079.

PIOVESAN, A.R.; MARTINELLI, A.H.S.; LIGABUE-BRAUN, R.; SCHWARTZ, J.-L.; CARLINI, C.R. Canavalia ensiformis urease, Jaburetox and derived peptides form ion channels in planar lipid bilayers. Archives of Biochemistry and Biophysics, v.547, p.6-17, 2014. DOI: 10.1016/j.abb.2014.02.006.

POLYORACH, S.; WANAPAT, M. Improving the quality of rice straw by urea and calcium hydroxide on rumen ecology, microbial protein synthesis in beef cattle. Journal of Animal Physiology and Animal Nutrition, v.99, p.449-456, 2015. DOI: 10.1111/ jpn.12253.
RAMÍREZ, G.R.; AGUILERA-GONZÁLEZ, J.C.; GARCÍADÍAZ, G.; NÚÑÉS-GONZÁLES, A.M. Effect of urea treatment on chemical composition and digestion of Cenchrus ciliaris and Cynodon dactylon hays and Zea mays residues. Journal of Animal and Veterinary Advances, v. 6, p.1036-1041, 2007.

REIS, R.A.; RODRIGUES, L.R. de A.; RESENDE, K.T. de; PEREIRA, J.R.A.; RUGGIERI, A.C. Avaliação de fontes de amônia para o tratamento de fenos de gramíneas tropicais: 2 . Compostos nitrogenados. Revista Brasileira de Zootecnia, v.30, p.682-686, 2001. DOI: 10.1590/S1516-35982001000300011.

SARMENTO, P.; GARCIA, R.; PIRES, A.J.V.; NASCIMENTO, A.S. Grãos de soja como fonte de urease na amonização do bagaço de cana-de-açúcar com uréia. Scientia Agricola, v.58, p.223-227, 2001. DOI: 10.1590/S0103-90162001000200002.

SILVA, D.J.; QUEIROZ, A.C. de. Análise de alimentos: métodos químicos e biológicos. 3.ed. Viçosa: Ed. da UFV, 2002. 235p.

SNIFFEN, C.J.; O'CONNOR, J.D.; VAN SOEST, P.J.; FOX, D.G.; RUSSELL, J.B. A net carbohydrate and protein system for evaluating cattle diets: II, Carbohydrate and protein availability. Journal of Animal Science, v.70, p.3562-3577, 1992. DOI: $10.2527 / 1992.70113562 x$.

VADIVELOO, J.; FADEL, J.G. The response of rice straw varieties to urea treatment. Animal Feed Science and Technology, v.151, p.291-298, 2009. DOI: 10.1016/j.anifeedsci.2009.03.003.

VAN SOEST, P.J.; ROBERTSON, J.B.; LEWIS, B.A. Methods for dietary fiber, neutral detergent fiber, and nonstarch polysaccharides in relation to animal nutrition. Journal of Dairy Science, v.74, p.3583-3597, 1991. DOI: 10.3168/jds.S0022-0302(91)78551-2.

VELÁSQUEZ, P.A.T.; BERCHIELLI, T.T.; REIS, R.A.; RIVERA, A.R.; DIAN, P.H.M.; TEIXEIRA, I.A.M. de A. Composição química, fracionamento de carboidratos e proteínas e digestibilidade in vitro de forrageiras tropicais em diferentes idades de corte. Revista Brasileira de Zootecnia, v.39, p.12061213, 2010. DOI: 10.1590/S1516-35982010000600007.

VIEIRA, R.A.M.; TEDESCHI, L.O.; CANNAS, A. A generalized compartmental model to estimate the fibre mass in the ruminoreticulum: 1. Estimating parameters of digestion. Journal of Theoretical Biology, v.255, p.345-356, 2008. DOI: 10.1016/j. jtbi.2008.08.014.

WANAPAT, M.; POLYORACH, S.; BOONNOP, K.; MAPATO, C.; CHERDTHONG, A. Effects of treating rice straw with urea or urea and calcium hydroxide upon intake, digestibility, rumen fermentation and milk yield of dairy cows. Livestock Science, v.125, p.238-243, 2009.

Received on August 8, 2016 and accepted on March 31, 2017

Pesq. agropec. bras., Brasília, v.52, n.12, p.1268-1275, dez. 2017 DOI: $10.1590 / \mathrm{S} 0100-204 \mathrm{X} 2017001200016$ 